\title{
An Enhanced Technique for Exemplar based Image Inpainting
}

\author{
Jaspreet Kaur Chhabra \\ Department of Computer Science \& Engg \\ S.V.C.E \\ Indore, M.P, India
}

\author{
Vijay Birchha \\ Department of Computer Science \& Engg \\ S.V.C.E \\ Indore, M.P, India
}

\begin{abstract}
Image inpainting is an emerging area of image processing; with the help of this we can fill the misplaced or lost regions of a given image. In real life for example in the museum world the job of inpainting of precious images or paintings is fulfilled with the help of an expert of arts. Like this in the digital world the job of image inpainting is fulfilled with the help of inpainting algorithms. Various algorithms have presented in the past to achieve the task of image inpainting. In this paper, we have suggested a novel exemplar based image inpainting technique. The suggested technique will remove whole object from a given image or a portion of the object \& it will offer high quality outcomes.
\end{abstract}

\section{Keywords}

Image Inpainting, Exemplar Based Image Inpainting, Object Removal, and Filling Area.

\section{INTRODUCTION}

Recently, image inpainting techniques have achieved high reputation in the area of digital image processing. The techniques that are utilized for repairing the lost part of a picture or image are known as image inpainting technique. The application area of image inpainting techniques is very large; these techniques are utilized for- elimination of any object or anything from a given image, reinstallation of longstanding pictures/images, deletion of blurs of dust in picture/image, improvement of red-eye, inventive effect by deleting objects etc. Generally, we have seen two categories of procedures in literature that deal with the problems related to image inpainting and which are described above and those procedures are based on- texture synthesis and image inpainting. The procedures which are utilized to produce large scale image regions with the help of sample textures are called texture synthesis methods, these types of methods mainly concentrated on texture part of image. The procedures which are utilized to fill up the little gap in image are called inpainting methods, these types of methods mainly concentrated on structural part of picture/image [1][3].

A new type of image inpainting method recognized as exemplar based image inpainting method have proposed in literature, which employ both texture synthesis technique as well as inpainting technique. So, we can recreate both part of an image texture part and also structural part with the help of exemplar based image inpainting technique [2][3].

In literature we have studied various types of image inpainting approaches, for example- PDE based image inpainting approaches, Exemplar based image inpainting techniques, Texture Synthesis based image inpainting methods, Semi- automatic \& Fast Inpainting methods and Hybrid inpainting methods.
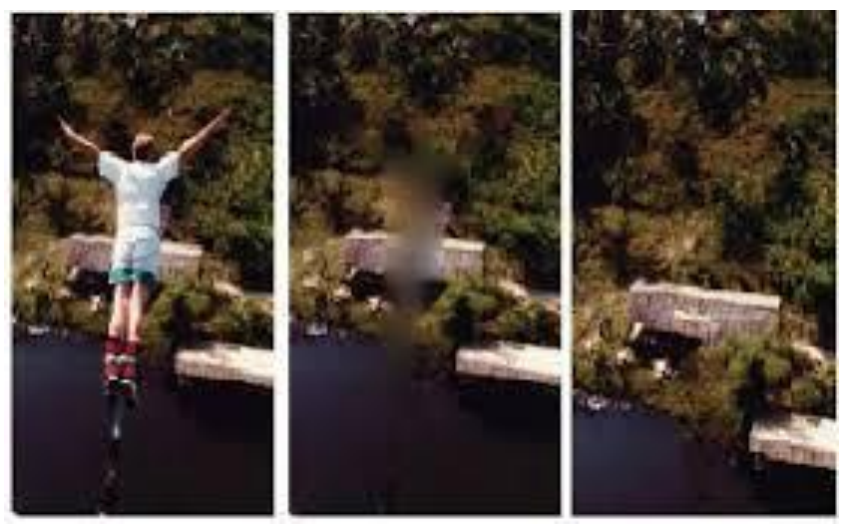

Figure 1: Example of Image Inpainting

Figure-1 shows the example of image inpainting, in which the picture is divided into three parts. The first part shows the original picture, from which we want to remove the photo of man and restore the picture without man. The intermediate part shows the picture without man but with some distortion. Part three shows that the final required picture without man and without distortion. Here, part two and three required image inpainting.

The exemplar based image inpainting technique efficiently recreates an image with large object or target regions. There are two steps which are utilized by exemplar based image inpainting technique- at first the priority assignment is prepared and second select the best matching patch from image [3].

The exemplar based image inpainting technique choose the most similar patches from the area of image to be inpaint, then the matching of these most similar patches is computed by certain metrics, and finally the missing area is fill up by most similar patches. On the basis of filling order, this method fill up the structures in the absent regions by utilizing the spatial information/ details of nearby regions [1] [4].

In this paper, we have suggested a novel exemplar based image inpainting technique. The suggested technique will remove whole object from a given image or a portion of the object \& it will offer high quality outcomes.

\section{LITERATURE SURVEY}

Various researchers have suggestedtheir work in theareaof image inpainting particularly in exemplar based image 
inpainting; some of them which is mostimportantare revealed in this section.

\subsection{A. Criminisi, P. Perez and K. Toyama [1]}

In this paper authors have offered a novel technique which eliminates enormous objects from a given image. The problem of elimination of enormous objects from a given image has been solved with the help of two categories of techniquestexture synthesis techniques and inpainting technique. They have proposed an efficient and innovative technique that takes benefits of both techniques texture synthesis and inpainting. They have suggested a best first method wherein the order of filling is very essential feature for image renovation and the proficiency of this method is normally trusting on this feature. The confidence value in the manufactured pixel values is broadcasted in a fashion analogous to the broadcast of info in inpainting technique.

\subsection{W. H. Cheng et al [5]}

In this paper authors have offered a novel strong algorithm for exampler based inpainting, which can be altered to any given image substances of different features. Authors have offered a common priority function that properly incorporates both the texture information and structure information for to simplify the process of image renovation, this is the key contribution of this research work. With the help of exemplar examination and component weight choice ruled the computational complexity of the suggested technique.

\subsection{K. Sangeetha, P. Sengottuvelan and E. Balamurugan [6]}

In this paper authors have offered a novel exemplar based image inpainting technique with an enhanced priority factor that expresses the filling order of patches in the given image. Technique proposed by authors is dependent on the fact that the technique broadcast the patch by means of silently broadcasting the image patches from the source area into the inward of the target area patch by patch. They were demonstrated that the suggested exemplar based inpainting technique achieves better results in terms of PSNR (peak signal to noise ratio) as compared to other existing image inpainting techniques.

\subsection{Waykule J. M. [7]}

In this paper authors have suggested a new technique by adapting the present exemplar based inpainting technique and make it highly appropriate for eliminating big objects from given images. The main aim of inpainting techniques is to achieve quality of filling and it is highly dependent on the processing time of these techniques. The key emphasis of this technique is to produce better outcomes as compared to the existing image inpainting techniques and for this purpose they improve the priority function. They have proposed an efficient and innovative technique that takes benefits of both techniques texture synthesis and inpainting. They were demonstrated that the suggested exemplar based inpainting technique achieves better outcomes and it reduced the time of inpainting the target area up to one fourth as compared to other existing image inpainting techniques.

\subsection{Sreelekshmi Das and Reeba R. [8]}

In this paper authors have demonstrated an exemplar based video inpainting technique that reestablishes the portion of the deleted object, and additionally this technique can be utilized to excerpt the background information of given videos. The inpainting technique proposed in this paper initially utilizes standardized cross correlation to transform the video into the frames and the object tracing in every frame. The lost part of given video is then iteratively determine in the source area with the help of exemplar based inpainting technique and the most analogous patch is filled in the lost/target part. To enhance the priority factor that expresses the filling order of patches in the given image and to diminish the search area the offered technique utilize segmentation map method which routinely choose the values of these parameters.

In this paper, we have suggested a novel exemplar based image inpainting technique. The suggested technique will remove whole object from a given image or a portion of the object \& it will offer high quality outcomes.

\section{PROPOSED METHOD}

In this section, we will describe the offered technique by which we can gain our proposed objectives. The outline of the planned technique is as follows:

\subsection{Selection of target region}

Initially, user chooses the region which wants to be inpainted; this is done with the help of other image processing tools because it is separate process then inpainting. In general, for the choice of the target region, we utilize a specific color for example- green. In addition to this, there must be no loss of generality.

\subsection{Locate all the borders of the target region}

After the selection of the region which wants to be inpainted; we locate all the boundaries/borders of the target area and this is done with the help of other image processing tools, because our aim is just to renovate the given image.

\subsection{In the target area, choose a patch which is to be in-painted}

Now, in the target region, we choose a patch which is to be inpainted. The patch size is generally chosen in such a way that it should be large than the biggest distinguishable region of the image. In our method, the default patch size we have utilized is $7 * 7$. It can be changed. Also the patch is indicated by PTCH.

\subsection{Find out the similar patch in the image}

Now we can search the similar patch in the image. We utilize the Mean Squared Error to discover the best similar patch.

$$
\text { MSE }=\sum \frac{\left(f_{x, y}-g_{x, y}\right)^{2}}{N} .
$$

\subsection{Inpaint the image}

Now we can inpaint the image with the help of patch discovered in the previous step. 


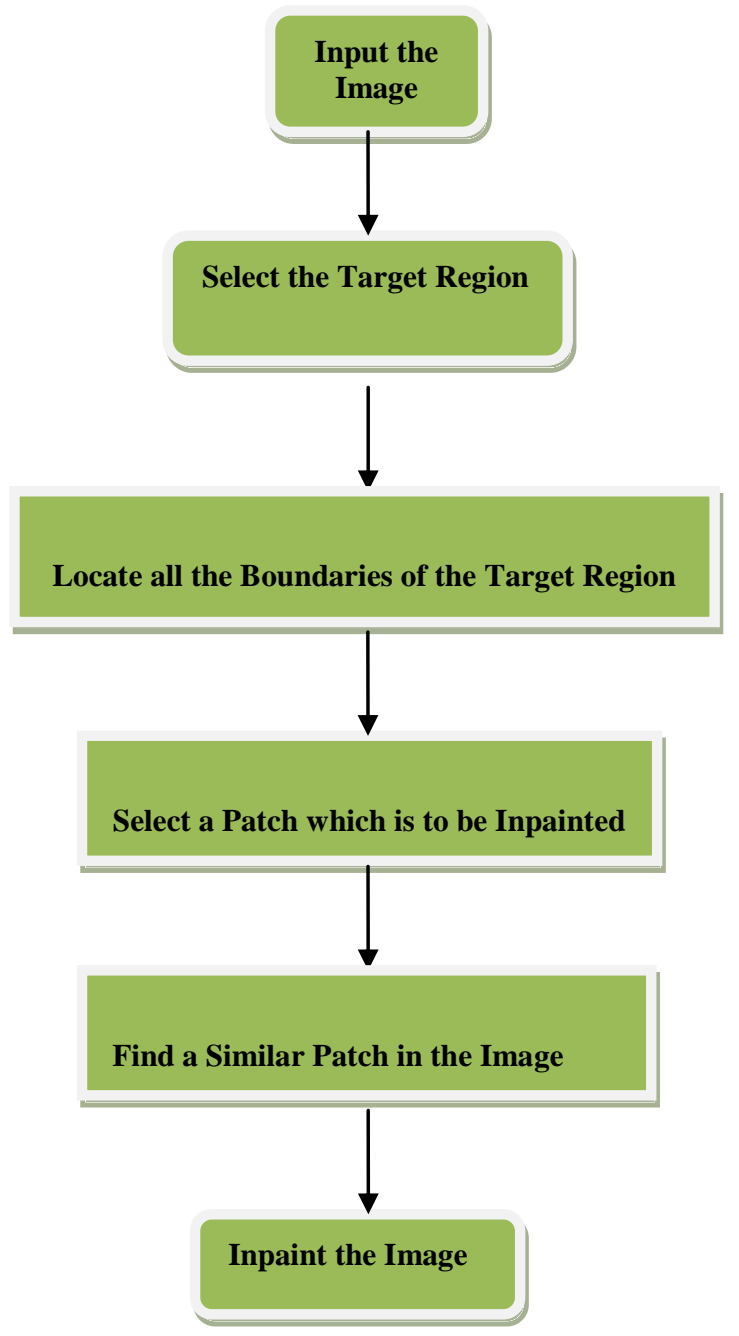

Figure 2: Shows the Outline of Proposed Model for Image Inpainting

\section{EXPERIMENTAL RESULTS}

For this research work, we have utilized various images likeboat image, hill image, lamp image, sea land Image etc. We can enter an image with some specific file formats like- jpg, jpeg, png, bmp and gif, proposed system not allowed an image other than these file formats. All the experiments were performed with the help of Net Beans IDE 7.3.1. Figure-3 shows some examples of input images. As the proposed technique is already been discussed in previous section and the proposed model is shown in figure-2, so, initially we input the image. After the selection of input image we select the target region and locate all the boundaries/borders of the target area and this is done with the help of other image processing tools, because our aim is just to renovate the given image. Now with the help of proposed technique we choose a patch which is to be in-painted and find out the similar patch in the image. Now we can inpaint the image with the help of patch discovered.

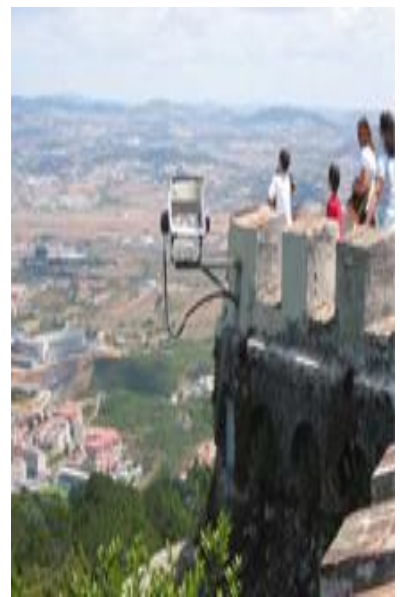

(a) Hill Image

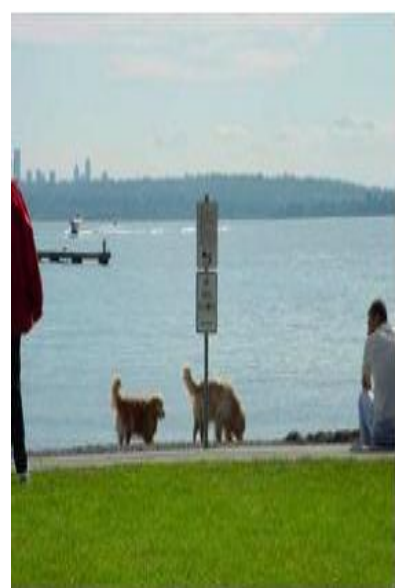

(c) Sea Land Image

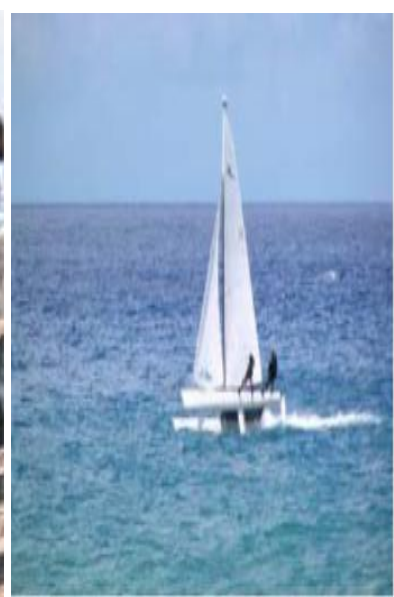

(b) Boat Image

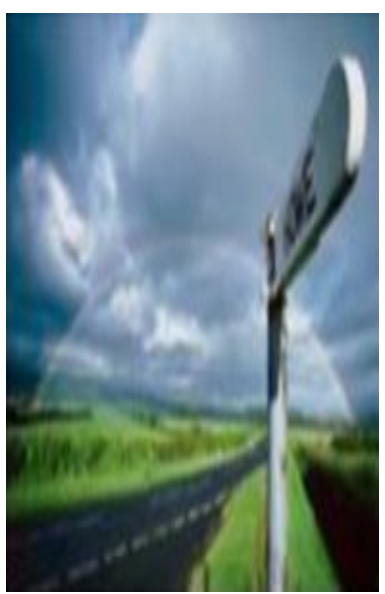

(d) Lamp image
Figure 3: Example of input images

The experimental results are shown in Snapshot- 4, 5, 6 and 7. The Snapshot-4 shows that the front page with control menu for inpainting, like- file menu (open image, save, save as), edit menu (undo, redo), Inpaint (run the algorithm). Snapshot-5 shows that the source/input image which want to be renovate. Snapshot-6 shows the selection of initial mask from source image which want to be renovate. Snapshot-7 shows that the resultant renovated image. 


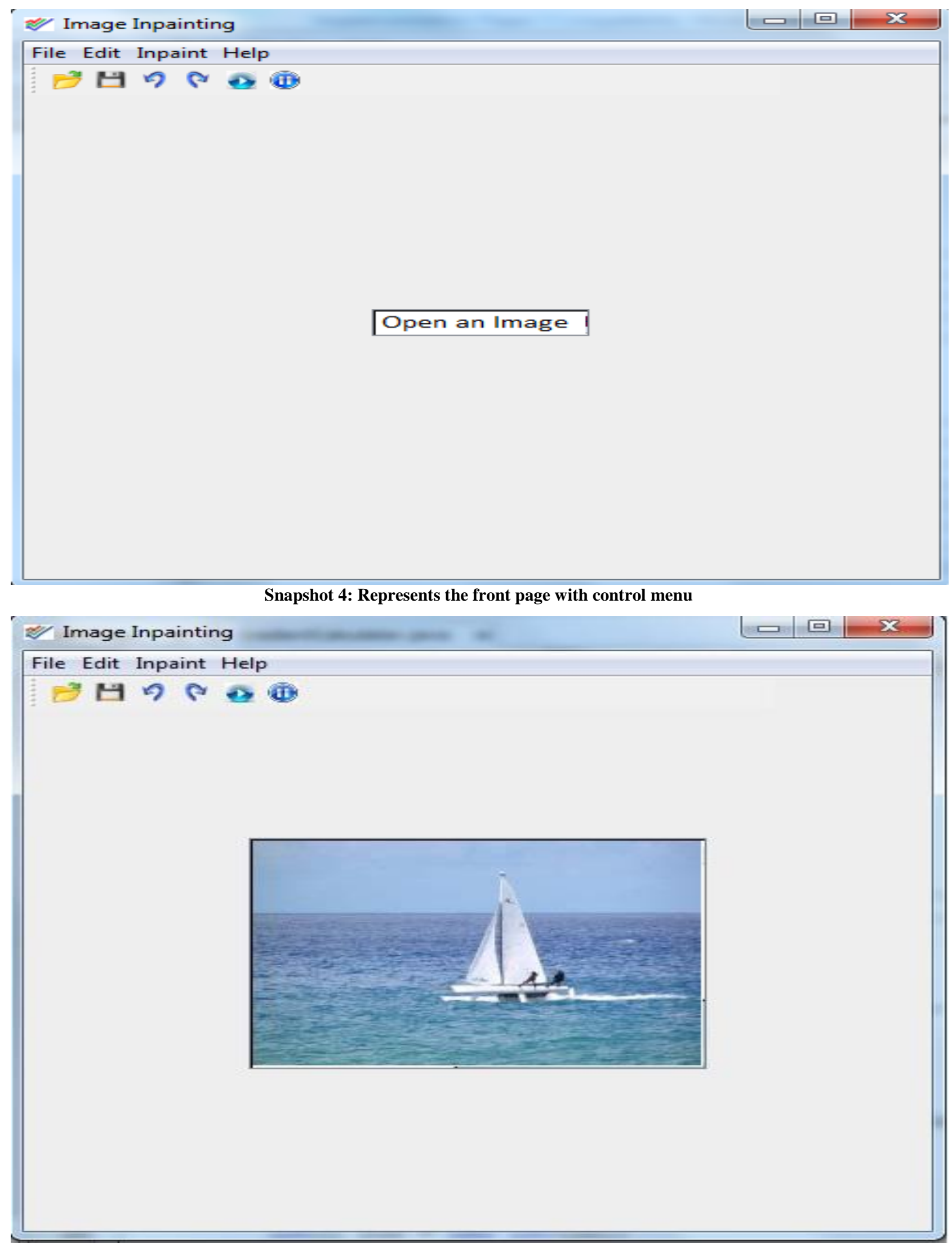

Snapshot 5: Represents the image entered by the user 


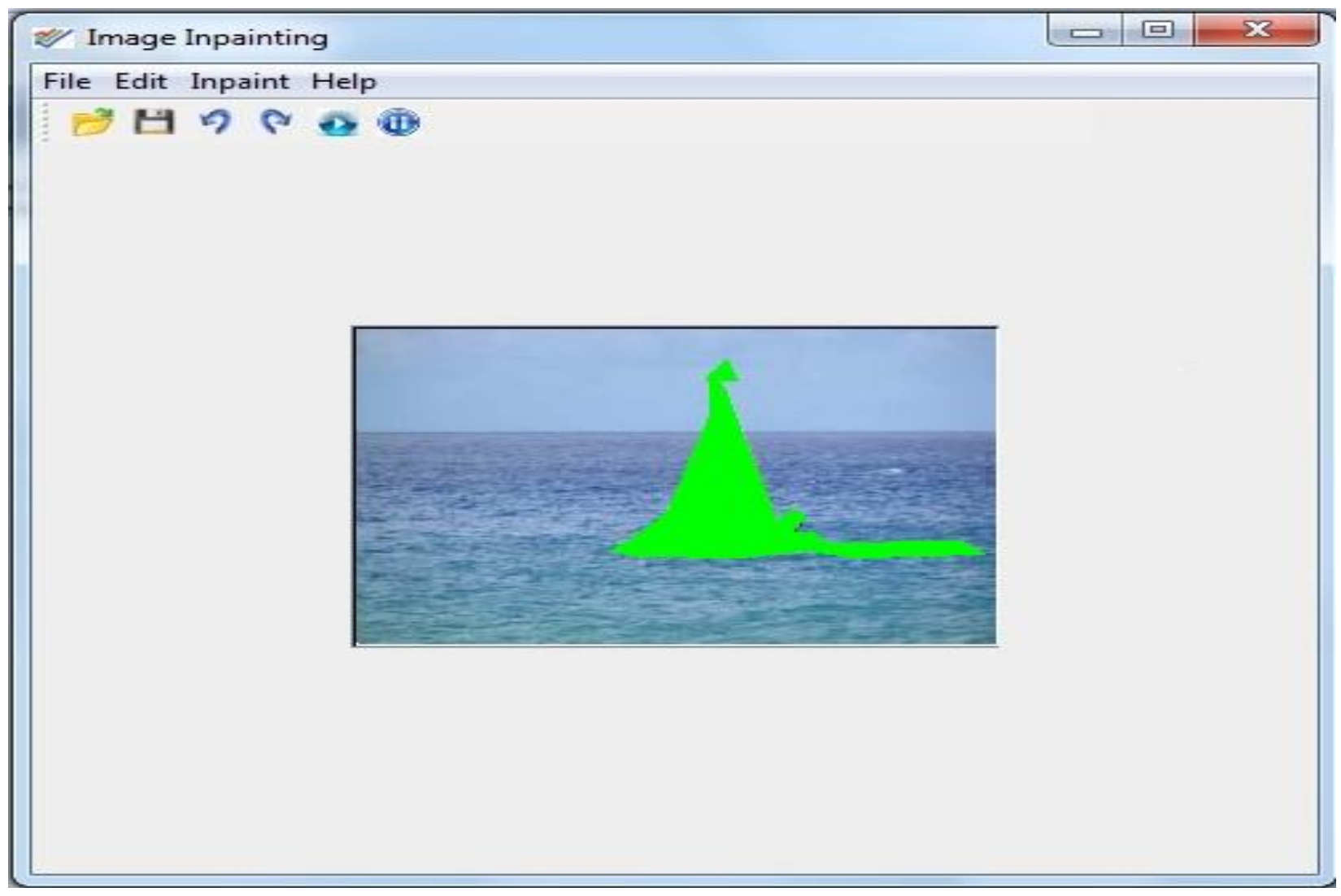

Snapshot 6: Represents the target area located with green color

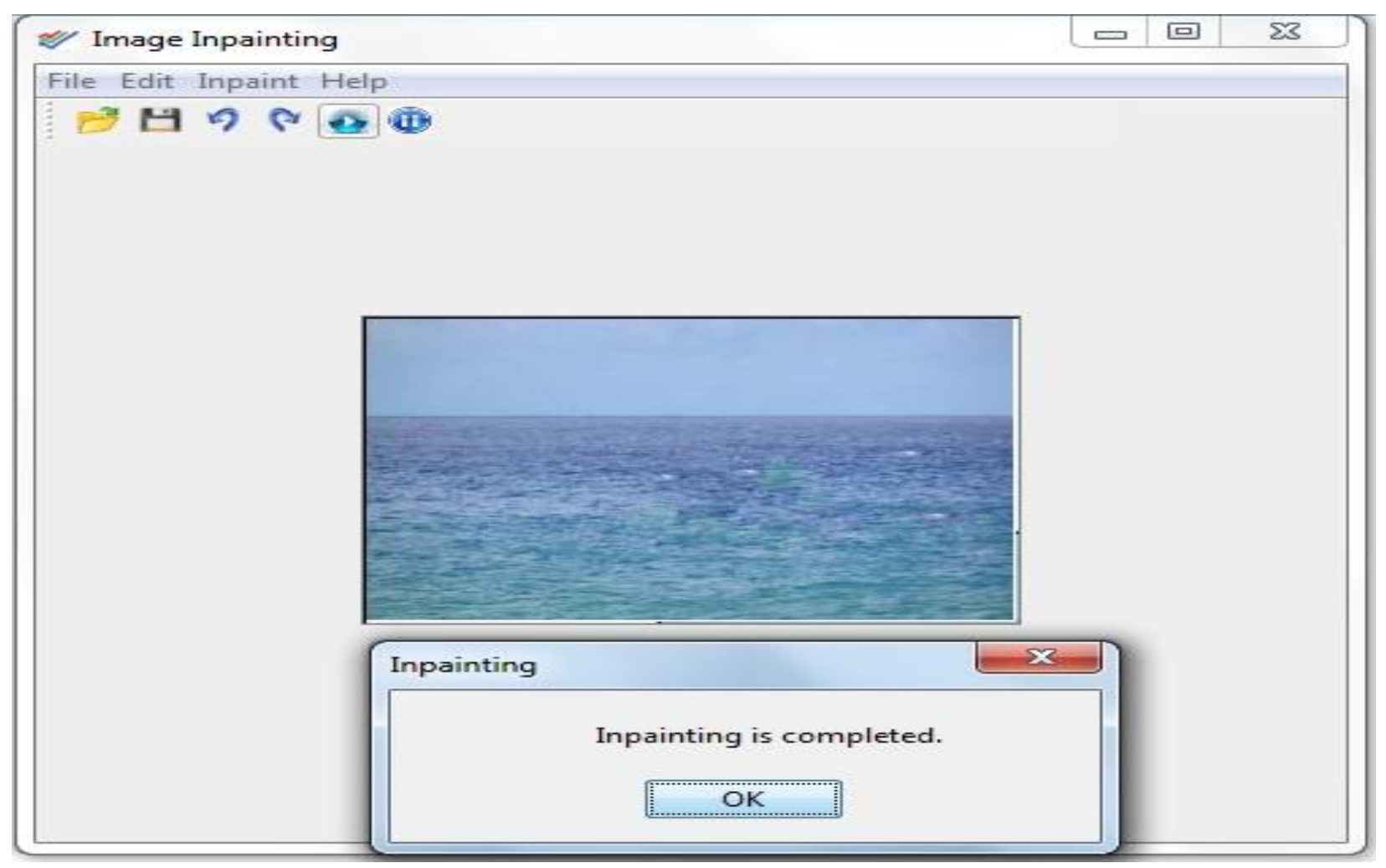

Snapshot 7: Represents the inpainted image 
The comparison of efficiency/time of proposed system with the existing exemplar based inpainting and divided image in exemplar based inpainting is shown in table- 1. As shown in table-1 we utilized three images for inpainting purposes hill image, boat image, and lamp image, sea land image as shown in figure- 3.

Table 1: Comparison of efficiency/time of proposed system with the existing exemplar based inpainting and divided image in exemplar based inpainting

\begin{tabular}{|c|c|c|c|c|}
\hline Images & $\begin{array}{c}\text { Size of } \\
\text { image }\end{array}$ & $\begin{array}{c}\text { Exemplar } \\
\text { Based } \\
\text { inpainting } \\
\text { (sec) }\end{array}$ & $\begin{array}{c}\text { Divided } \\
\text { image in } \\
\text { Exemplar } \\
\text { Based } \\
\text { inpainting } \\
\text { (sec) }\end{array}$ & $\begin{array}{c}\text { Proposed } \\
\text { Technique } \\
\text { (sec) }\end{array}$ \\
\hline $\begin{array}{c}\text { Hill } \\
\text { image }\end{array}$ & $215 \times 187$ & 35.32 & 8.95 & 5.32 \\
\hline $\begin{array}{c}\text { Boat } \\
\text { Image }\end{array}$ & $342 \times 257$ & 431.92 & 108.67 & 55.64 \\
\hline $\begin{array}{c}\text { Sea } \\
\text { land } \\
\text { Image }\end{array}$ & $279 \times 210$ & 178.45 & 46.40 & 11.52 \\
\hline $\begin{array}{c}\text { Lamp } \\
\text { Image }\end{array}$ & $216 \times 143$ & 46.26 & 10.89 & 4.46 \\
\hline
\end{tabular}

Figure 8: shows the comparison graph for efficiency/time of existing exemplar based inpainting and divided image in exemplar based inpainting. As exposed in figure 8 the proposed image inpainting system performs better as compared to the existing image inpainting techniquesexemplar based inpainting technique and divided image in exemplar based inpainting technique.

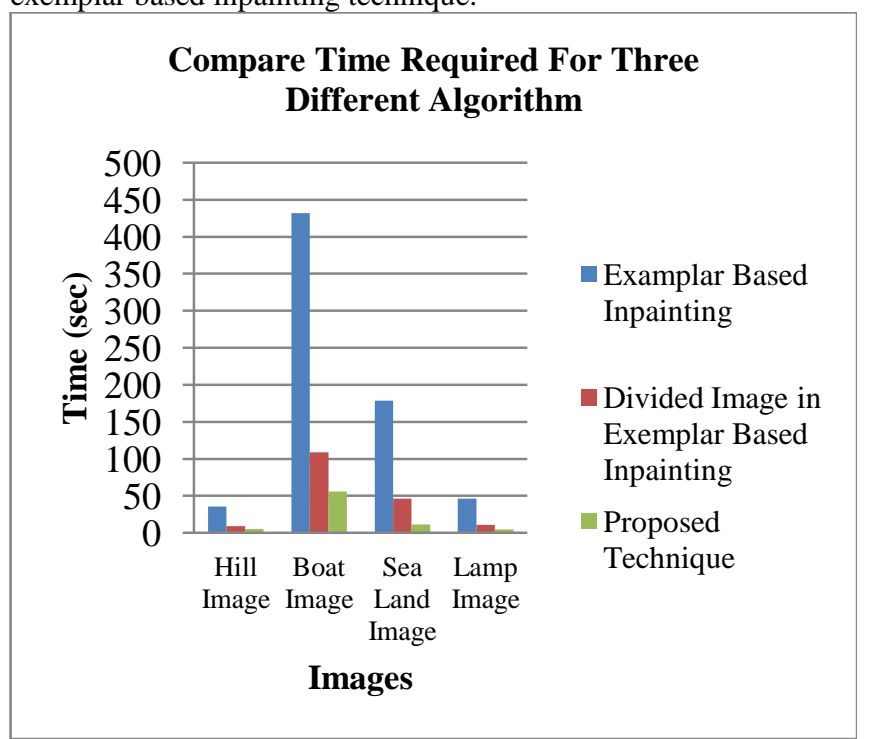

Figure 8: Comparison of efficiency/time of proposed system with the existing exemplar based inpainting and divided image in exemplar based inpainting

\section{CONCLUSION}

Recently, image inpainting techniques have achieved high reputation in the area of digital image processing. The techniques that are utilized for repairing the lost part of a picture or image are known as image inpainting technique. In this paper, we have suggested an innovative exemplar based image inpainting technique. The suggested technique will remove whole object from a given image or a portion of the object \& the experimental outcomes shows that it offers high quality inpainted image. In current research work we have taken the fixed patch size $(7 * 7)$, in near future we will work on different size patches. Current research work is only concentrate on the image inpainting; in near future we will implement it for video inpainting.

\section{REFERENCES}

[1] A. Criminisi, P. Perez and K. Toyama, "Region Filling and Object Removal by Exemplar Based Image Inpainting", IEEE Transactions on Image Processing, 13(9), pp. 1200-1212, 2004

[2] Jayesh Patel and Tanuja K. Sarode, "Exemplar based Image Inpainting with Reduced Search Region", International Journal of Computer Application, Vol. 92 No.12, April 2014

[3] Jaspreet Kaur Chhabra and Mr. Vijay Birchha, "Detailed Survey on Exemplar Based Image Inpainting Techniques", International Journal of Computer Science and Information Technologies, Vol. 5 (5), 2014.

[4] Komal S. Mahajan and Prof. M. B. Vaidya, "Image in Painting Techniques: A survey", IOSR Journal of Computer Engineering (IOSRJCE), ISSN: 2278-0661, ISBN: 2278-8727 Volume 5, Issue 4, PP 45-49, Sep-Oct. 2012.

[5] W. H. Cheng, C. W. Hsieh, S. K. Lin, C. W. Wang, and J. L. Wu, "Robust algorithm for exemplar-based image inpainting", Processing of International Conference on Computer Graphics, Imaging and Vision 2005, pp. 64 69, Beijing, China, Jul. 2005.

[6] K. Sangeetha, P. Sengottuvelan and E. Balamurugan , "A Novel Exemplar based Image Inpainting Algorithm for Natural Scene Image Completion with Improved Patch Prioritizing", International Journal of Computer Applications (0975 - 8887), Volume 36- No.4 December 2011.

[7] Waykule J.M., "Modified Image Exemplar-Based Inpainting", International Journal of Advanced Research in Computer and Communication Engineering,Vol. 2, Issue 9, September 2013.

[8] Sreelekshmi Das and Reeba R., "Robust Exemplar based Object Removal in Video", International Journal of Scientific Engineering and Research (IJSER), ISSN (Online): 2347-3878, Volume 1 Issue 2, October 2013. 